\title{
Nutrition, Food and Water Contaminants
}

National Cancer Institute

\section{Source}

National Cancer Institute. Nutrition, Food and Water Contaminants. NCI Thesaurus.

Code C15437.

Role of food and water contaminants in cancer causation or prevention and in general health. 